\title{
Comparison of BK Virus Nephropathy Risk Between Double-J-Stent with Anti-Reflux Mechanism and Standart Double-J-Stent: Single-Center Experience
}

\author{
nurettin ay ${ }^{1}$, Ramazan Danis ${ }^{2}$, seyhmus kaya ${ }^{2}$, mehtap akbalik kaya ${ }^{2}$, and aysel taktak ${ }^{2}$ \\ ${ }^{1}$ Diyarbakir Gazi Yasargil Training and Research Hospital \\ ${ }^{2}$ Affiliation not available
}

July 17, 2021

\begin{abstract}
Objectives: Ureteral stend use is a risk factor for BK nephropathy (BKVN). In 2015, we compared the patients with antireflux mechanism DJS (ARD-DJS) and those used standard DJS (st-DJS) in terms of BKV and BKVN frequency in 90 kidney transplant patients in two centers. With the increase in the number of our patients over time and lengthening of the follow-up duration, we needed to re-evaluate the data in one center. Materials and Methods: We retrospectively evaluated 211 patients who underwent kidney transplantation at Diyarbakır Gazi Yaşargil Training and Research Hospital between September 2012 and September 2019. The following parameters were recorded, demographic data, immunosuppression protocols, presence of rejection, graft loss, plasma BKV levels, and presence of BKVN. Median and IQR follow-up time for ARD-DJS and St-DJS patients was 72 months (62,5-80,3 months) and 27,8 months (17,4-39,6 months) respectively. Results: Thirteen patients (6,1\%) had BKV viremia. BKVN was revealed by kidney biopsy in 3 of 13 patients. However, graft loss due to BKVN was observed in only one patient. ARD-DJS was used in 4 of these cases and standard DJS was used in 9 of these cases. Patients in whom BKV revealed in the first 3 months were compared in the aspect of DJS technique, BKV was significantly less observed in the ARD-DJS group (ARD-DJS: 2 patients; St-DJS:9 patients), ( $\mathrm{p}=0,046)$. Conclusions: In our study, BKV was observed less in patients with ARD-DJS that were clinically significant but not statistically significant. Therefore, prospective randomized studies with high patient numbers are needed to determine the effectiveness of ARD-DJS.
\end{abstract}

Comparison of BK Virus Nephropathy Risk Between Double-J-Stent with Anti-Reflux Mechanism and Standart Double-J-Stent: Single-Center Experience

\section{Abstract}

Objectives: Ureteral stend use is a risk factor for BK nephropathy (BKVN). In 2015, we compared the patients with anti-reflux mechanism DJS (ARD-DJS) and those used standard DJS (st-DJS) in terms of BKV and BKVN frequency in 90 kidney transplant patients in two centers. With the increase in the number of our patients over time and lengthening of the follow-up duration, we needed to re-evaluate the data in one center.

Materials and Methods: We retrospectively evaluated 211 patients who underwent kidney transplantation at Diyarbakır Gazi Yaşargil Training and Research Hospital between September 2012 and September 2019. The following parameters were recorded, demographic data, immunosuppression protocols, presence of rejection, graft loss, plasma BKV levels, and presence of BKVN.

Median and IQR follow-up time for ARD-DJS and St-DJS patients was 72 months (62,5-80,3 months) and 27,8 months (17,4-39,6 months) respectively. 
Results: Thirteen patients $(6,1 \%)$ had BKV viremia. BKVN was revealed by kidney biopsy in 3 of 13 patients. However, graft loss due to BKVN was observed in only one patient. ARD-DJS was used in 4 of these cases and standard DJS was used in 9 of these cases. Patients in whom BKV revealed in the first 3 months were compared in the aspect of DJS technique, BKV was significantly less observed in the ARD-DJS group (ARD-DJS: 2 patients; St-DJS:9 patients), $(\mathrm{p}=0,046)$.

Conclusions: In our study, BKV was observed less in patients with ARD-DJS that were clinically significant but not statistically significant. Therefore, prospective randomized studies with high patient numbers are needed to determine the effectiveness of ARD-DJS.

\section{Keywords: BKV, kidney transplantation, ureteral stend}

\section{What is already known about this topic?}

Risk factors for BKV are aggressive immunosuppression, high human leukocyte antigen (HLA) mismatch, ureteral stent use, rejection therapy, prolonged ischemia duration, advanced age, male recipient, diabetes mellitus (DM), lymphocyte depleting induction therapies, tacrolimus, and mycophenolate- based regimens.

\section{What does this article add?}

In our study, BKV was observed less in patients with ARD-DJS that were clinically significant but not statistically significant. Also, it was observed that the majority of patients with early developed BKV (first 3 months) were patients with st-DJS. Although close monitoring and reduction of immunosuppression prevent BKVN to a great extent, all arguments that can reduce the risk of BKV deserve to be investigated in detail.

\section{Introduction}

BK virus (BKV) is a member of the polyomavirus family, which was first isolated in 1971 from the urine sample of a renal transplant patient, with clinical observations made over the years, it was understood that BKV is responsible for BKV nephropathy (BKVN) (1-3). The exact prevalence of BKVN is not known, however, it is estimated to be in the range of $1-15 \%$. BKVN is an important clinical problem causing graft loss in 15-50\% of transplant patients (4). Risk factors for BKV are aggressive immunosuppression, high human leukocyte antigen (HLA) mismatch, ureteral stent use, rejection therapy, prolonged ischemia duration, advanced age, male recipient, diabetes mellitus (DM), lymphocyte depleting induction therapies, tacrolimus, and mycophenolate- based regimens (5-10). It has been shown that the use of double-J-Stent (DJS) in experimental animal models causes superficial epithelial destruction, ulceration, and inflammatory changes on the transitional epithelium in rat ureters, as well as increasing the incidence of BKVN by 4 times (2,11-13). Studies have shown that conditions that cause persistent hydronephroses, such as posttransplant ureter flexion and ureteral stenosis, may play a dynamic role in the pathogenesis of polyoma virus-associated nephropathy by causing ureteral reflux. Vesicoureteral reflux (VUR) may occur with normal bladder contraction while using DJS $(14,15)$. In 2015, we compared the patients with anti-reflux mechanism DJS (ARD-DJS) and those used standard DJS (st-DJS) in terms of BKV and BKVN frequency in 90 kidney transplant patients in two centers (Figure 1). In this study, BKV was observed with significantly less frequency in univariate analyzes in patients with ARD-DJS. However, no significant difference was observed in the multivariate analyzes. In this study, the small number of cases and the short follow-up duration were the factors limiting our study (13). With the increase in the number of our patients over time and lengthening of the follow-up duration, we needed to re-evaluate the data in one center.

\section{Materials and Methods}

We retrospectively evaluated 211 patients who underwent kidney transplantation at Diyarbakır Gazi Yaşargil Training and Research Hospital between September 2012 and September 2019. One hundred seventy of the patients had a living donor and 41 of them had deceased donor kidney transplantation. The following parameters were recorded, demographic data, immunosuppression protocols, presence of rejection, graft loss, plasma BKV levels, and presence of BKVN. (Table 1,2) 
The study was conducted under the principles of the 2008 Helsinki Declaration. Informed consent was obtained from all the patients.

\section{Immunosuppression and Prophylaxis}

Basiliximab (20 mg at days 0 and 4 of operation) or anti-thymocyte globulin (ATG; for high-risk patients, $3 \mathrm{mg} / \mathrm{kg}$ during operation and $1,5 \mathrm{mg} / \mathrm{kg}$ at postoperative days 1 and 2) were used as induction therapy. Methylprednisolone $1000 \mathrm{mg}$ was given intra-operatively. Methylprednisolone dose was decreased by half every day and $20 \mathrm{mg}$ oral prednisolone was started on the 6th postoperative day for daily use. Oral prednisolone dosage was reduced gradually to reach $5 \mathrm{mg}$ a day in the first year after transplantation. Calcineurin inhibitors (tacrolimus or cyclosporin) and mycophenolate mofetil (MMF; $2 \mathrm{~g}$ /day in two divided doses) or mycophenolate sodium (MMF; $1440 \mathrm{mg} /$ day, in two divided doses) were used as maintenance immunosuppression therapy. MMF was used as $600 \mathrm{mg} / \mathrm{m} 2$ in two divided doses in children. We considered both mycophenolate mofetil and mycophenolate sodium in doses described above as the same drugs in our study. Everolimus was used in only one case (plasma level of the drug was targeted as 8-10 mg/dl). Trimethoprim/sulfamethoxazole and valganciclovir (450 mg a day) was prescribed for Pneumocystis jirovecii and cytomegalovirus (CMV) prophylaxis for 6 months after the transplantation. Acute rejection was diagnosed by kidney biopsy. The acute cellular rejection was treated with intravenous pulse methylprednisolone or ATG depending on the severity of the rejection. Plasmapheresis was added for acute humoral rejection. Delayed graft function (DGF) was described as a need for hemodialysis in the first week of kidney transplantation.

\section{Surgical Technique}

An open or laparoscopic nephrectomy technique was applied for living donors. An extravesical technique (Lich-Gregoir) was applied to all transplanted patients for ureteroneocystostomy (UNS). Types of DJS used were dependent on the choice of surgeons. Cases were grouped later by whether their DJS had an anti-reflux mechanism or not (St-DJS vs ARD-DJS). Surgical drains were placed to all patients to the operation side. Urinary catheters were removed on the 5 th postoperative day. Surgical drains were removed if there were no urinary leak and after urinary catheters were removed. All DJSs were removed by cystoscopy under local anesthesia at the 4th postoperative week. Urinary complication was described as the presence of urinary leak and stenosis at UNS.

\section{Follow Up}

All patients were followed up closely for renal functions, BK viremia, and BKVN after kidney transplantation. First BKV tests were done during 1st postoperative month. All transplanted patients were tested for BKV from their serum by polymerase chain reaction (PCR) monthly in the first year after transplantation. All the patients were followed up at the posttransplant period for at least 12 months. Cases with $>500 \mathrm{copies} / \mathrm{ml}$ by two or more consecutive measurements were accepted as having viremia. The immunosuppression dose was reduced when there was a higher viremia load $(>5,000$ copies $/ \mathrm{ml})$. Firstly MMF was discontinued and the prednisolone dose was reduced. If viremia continued, the calcineurin dose was reduced. Leflunomide was used for patients with BKVN. Kidney biopsy was performed to all cases with viremia for excluding BKVN.

\section{Statistical Analysis}

Statistical analyses were performed using the SPSS software version 16. The variables were investigated using (histograms, probability plots) and analytical methods (Kolmogorov-Smirnov/Shapiro-Wilk's test) to determine whether or not they were normally distributed. Descriptive analyses were presented using medians and interquartile range (IQR) for the non-normally distributed and ordinal variables. The proportions of patients with ATG use, preemptive transplantation, and BKVN were grouped by types of DJS using cross-tabulations. The Chi-square test or Fisher's exact test, where appropriate, were used to compare these proportions in different groups. Blood creatinine measurements were not normally distributed; nonparametric tests were conducted to compare these parameters. The Mann-Whitney U test was used to compare the relationship between blood creatinine levels and the use of the ARD-DJS.

\section{Results}


In this study, the data of 211 patients who underwent kidney transplantation between September 2012 and September 2019 were retrospectively evaluated. The median and interquartile range (IQR) of age and time from kidney transplantation of patients was 32 years (22-42 years) and 48,9 months (26,2-71 months) respectively. Median and IQR follow-up time for ARD-DJS and St-DJS patients was 72 months $(62,5-80,3$ months) and 27,8 months (17,4-39,6 months) respectively.

Seventy patients $(33,1 \%)$ underwent preemptive kidney transplantation. Basiliximab was used in 66 patients $(31,3 \%)$ and ATG was used in 130 patients $(61,6 \%)$ as induction therapy. Fifteen patients $(7,1 \%)$ were not given induction therapy. Three cases used cyclosporin $+\mathrm{MMF}+$ prednisolone, eight cases used everolimus + tacrolimus + prednisolone, and all other patients used tacrolimus $+\mathrm{MMF}+$ prednisolone for maintenance immunosuppression. Acute rejection was observed in 13 patients, humoral rejection in 3 patients, humoral, and cellular rejection in 3 patients. In 6 patients, graft loss due to rejection was observed (CR:3 HR:2 CR+HR: 1 patient). In postoperative duration DGF was observed in 9 patients (4,2\%), graft loss was observed in 12 patients $(5,7 \%)$. DJS was used in all patients, ARD-DJS was used in 100 patients $(47,4)$. Thirteen patients $(6,1 \%)$ had BKV viremia. BKV was revealed by kidney biopsy in 3 of 13 patients. However, graft loss due to BKVN was observed in only one patient. Except for 2 patients viremia was observed in the first 3 months in these patients, while in the other 2 patients, viremia was observed at 12 and 84 months after transplantation respectively. These 2 patients were in the group of ARD-DJS, in 4 of 13 patients with BKV, ARD-DJS, and in 9 patients st-DJS was used. Patients in whom BKV revealed in the first 3 months were compared in the aspect of DJS technique, BKV was significantly less observed in the ARD-DJS group (ARD-DJS: 2 patients; St-DJS:9 patients), $(\mathrm{p}=0,046)$. St-DJS was used in 6 of 7 patients with viremia level $>10,000$, however, this finding was not statistically significant. When the patients were compared according to their final blood creatinine levels, the creatinine levels in patients with st-DJS and ARD-DJS was 1,19 (range 0,96-1,47) and 1,03 (range 0,86-1,27) respectively which was statistically significant $(\mathrm{p}=0,002)$. None of the patient groups had urinary leakage in the postoperative period. However, in one patient in each group, urinary stenosis was observed in the postoperative follow-up period and revision operation was performed.

\section{Discussion}

Ureteral stent use is considered as an independent risk factor for BKV viruria, BKV viremia, and BKVN. In a study conducted on 1147 patients, a ureteral stent was used in $443(38,6 \%)$ of the patients, and BKV was observed in $17,2 \%$ of these patients, on the other hand, this ratio was $13,5 \%$ in patients without stent (16). Moreno et al. (17) detected BKV in 11 patients (6\%) in their study conducted on 184 patients. In this study, they explained the low BKV rates by the different ureteral stent techniques. At the same time in this study, ureteral anastomosis was performed using the modified Taguchi extravesical reimplantation technique and, the stent was externalized from the skin with a $6 \mathrm{~F}$ radiopaque infant nasogastric tube, and the stent was removed on the 5th day (17). Gupta et al. (18) evaluated 402 transplant kidney biopsies between 2013 and 2016, BKVN was detected in $6(1,49)$ patients. They attributed the lower rate of BKVN than the literature to the low immunosuppression dose and the high match in transplants from live relative donors. In our study, BKV was observed in $13(6,1 \%)$ patients, which is lower than the rates of BKV in the literature. Another remarkable inference in our study is that BKV was observed in $9(8,1 \%)$ of 111 patients in which st-DJS was used and in $4 \%$ of the patients used ARD-DJS, although these rates are not statistically significant among themselves, they show that ureteral stenting technique and stent type may also have aclinical meaning. BKVN was observed in 3 patients in our study, one of these patients resulted in graft loss, and also diffuse deep vein thrombosis occurred in the same patient. This patient died due to diffuse pulmonary embolism during follow-up. Considering that acute venous thrombosis was observed in a patient diagnosed with BKVN in a case report published in 2015, it is important to pay attention to the relationship between BKVN and DVT despite case-level studies (19).

In our study, we also reached some results that we could not interpret, when we compared the final blood creatinine levels in both groups we revealed that the creatinine level was lower in the patients with ARD-DJS, additionally, although the patients with ARD-DJS had a much longer follow-up period, the number of graft 
loss was similar to the patients with st-DJS.

The limitations of this study were its retrospective nature, the absence of a control group without DJS, and the need for much higher patient numbers, although the number of patients increased than our previous study. However, to the best of our knowledge, there are not enough studies in the literature evaluating the relationship between ARD-DJS and BKV.

In our study, BKV was observed less in patients with ARD-DJS that were clinically significant but not statistically significant. Also, it was observed that the majority of patients with early developed BKV (first 3 months) were patients with st-DJS. Although close monitoring and reduction of immunosuppression prevent BKVN to a great extent, all arguments that can reduce the risk of BKV deserve to be investigated in detail. Therefore, prospective randomized studies with high patient numbers are needed to determine the effectiveness of ARD-DJS.

Disclosure of conflict of interest

None.

\section{References}

1. M Mengel, M Marwedel, J Radermacher et al: Incidence of polyomavirus-nephropathy in renal allografts: influence of modern immunosuppressive drugs. Nephrol Dial Transplant 18:1190, 2003. Doi: $10.1093 / \mathrm{ndt} / \mathrm{gfg} 072$

2. Thomas A, Dropulic L, Rahman M, Duvuru Geetha: Ureteral stents: a novel risk factor for polyomavirus nephropathy. Transplantation 84:433, 2007. Doi: 10.1097/01.tp.0000269616.21698.10

3. Pham PT, Schaenman J, Pham PC. BK virus infection following kidney transplantation: an overview of risk factors, screening strategies, and therapeutic interventions. Curr Opin Organ Transplant 2014;19:401e12. Doi: 10.1097/MOT.0000000000000101.

4. GP Balba, B Javaid, JG Timpone Jr. BK polyomavirus infection in the renal transplant recipient. Infect Dis Clin North Am 2013;27:271e83. Doi: 10.1016/j.idc.2013.02.002

5. J Gralla, J Huskey, AC Wiseman. Trends in immune function assay (ImmuKnow; Cylex) results in the first year post-transplant and relationship to BK virus infection. Nephrol Dial Transplant 2012; 27: 2565. Doi: $10.1093 / \mathrm{ndt} / \mathrm{gfr} 675$

6. DL Bohl, DC Brennan. BK virus nephropathy and kidney transplantation. Clin J Am Soc Nephrol 2007; 2 Suppl 1: S36. Doi: 10.2215/CJN.00920207

7. FE Yeo, CM Yuan, SJ Swanson et all. The prevalence of BK polyomavirus infec tion in outpatient kidney transplant recipients followed in a single center. Clin Transplant 2008; 22: 532-41. Doi: 10.1111/j.13990012.2008.00817.x

8. HH Hirsch, P Randhawa; AST Infectious Diseases Community of Practice: BK polyomavirus in solid organ transplantation. Am J Transplant 13[Suppl 4]: 179-188, 2013. doi: 10.1111/ctr.13528

9. JD Schold, S Rehman, LK Kayle, J Magliocca, TR Srinivas, HU Meier- Kriesche: Treatment for BK virus: Incidence, risk factors and outcomes for kidney transplant recipients in the United States. Transpl Int 22: 626-634, 2009. Doi: 10.1111/j.1432-2277.2009.00842.x

10. HH Hirsch, N Babel, P Comoli et all; ESCMID Study Group of Infection in Compromised Hosts: European perspective on human polyomavirus infection, replication and disease in solid organ transplantation. Clin Microbiol Infect 20[Suppl 7]: 74-88, 2014. Doi: 10.1111/1469-0691.12538

11. IA Atencio, FF Shadan, XJ Zhou, ND Vaziri, LP Villarreal. Adult mouse kidneys become permissive to acute polyomavirus infection and reactivate persistent infections in response to cellular damage and regeneration. J Virol 1993; 67: 1424-32. Doi: 10.1128/JVI.67.3.1424-1432.1993 
12. IA Atencio, LP Villarreal. Polyomavirus replicates in differentiating but not in proliferating tubules of adult mouse polycystic kidneys. Virology 1994; 201: 26-35. Doi: 10.1006/viro.1994.1262

13. Ay N, Bahadır MV, Anıl M et al. Comparison of anti-reflux mechanism between Double-J-Stent and standart Double-J-Stent use for risk of BK nephropathy and urinary tract Infection in kidney transplantation. Int J Clin Exp Med 2015;8(9):16340-16345

14. HA Mosli, HM Farsi, MF al-Zimaity, TR Saleh, MM al-Zamzami. Vesicoureteral reflux in patients with double pigtail stents. J Urol 1991; 146: 966-9. Doi: 10.1016/s0022-5347(17)37976-4.

15. L Kayle, I Zendejas, D Schain, J Magliocca. Ureteral stent placement and BK viremia in kidney transplant recipients. Transpl Infect Dis 2013; 15: 202-7. Doi: 10.1111/tid.12051

16. J G Maliakkal, D C. Brennan, C Goss et all. Ureteral Stent Placement and Immediate Graft Function are associated with Increased Risk of BK Viremia in the First Year after Kidney Transplantation. Transpl Int. 2017 February ; 30(2): 153-161. doi:10.1111/tri.12888. Doi: 10.1111/tri.12888

17. A I Martinez Moreno, J G Garcia, V R Mari, E P Ayala. Prevalence of BK Virus in Renal Transplant at a Single Center: Experience With Our Ureteral Reimplantation Surgical Technique. DOI: 10.6002 /ect.2019.0425

18. P Gupta, A Gupta, A. K. Bhalla et all. BK Virus Nephropathy in Living Donor Renal Allograft Recipients: An Observational Study from a Large Transplant Center in India. Saudi J Kidney Dis Transpl 2018;29(6):1366-1370. Doi: 10.4103/1319-2442.248313

19.D Pucar, K Klein, J Corley, H T Williams. BK Nephritis and Venous Thrombosis in Renal Transplant Recipient Detected by 111In Leukocyte Imaging. Clin Nucl Med 2015 Jul;40(7):e382-5.Doi: 10.1097/RLU.0000000000000813.

ARD-DJS: anti-reflux mechanism double J stenting; st-DJS: standart-DJS; ATG: anti thmocyte globülin DGF: delayed graft function; BKV: BK viremia; BKVAN: BKV-associated nephropathy; Cr: creatine.

Table 2: Comparison of BKV positive and negative cases

Donor Cadaveric Alive Preemptive Dialysis patient DGF (+) DGF (-) Mismatch 0 MM 1MM 2MM 3 MM 4 MM 5 MM 6 I

\section{Hosted file}

Table 1-2.docx available at https://authorea.com/users/419681/articles/530712-comparisonof-bk-virus-nephropathy-risk-between-double-j-stent-with-anti-reflux-mechanism-andstandart-double-j-stent-single-center-experience 


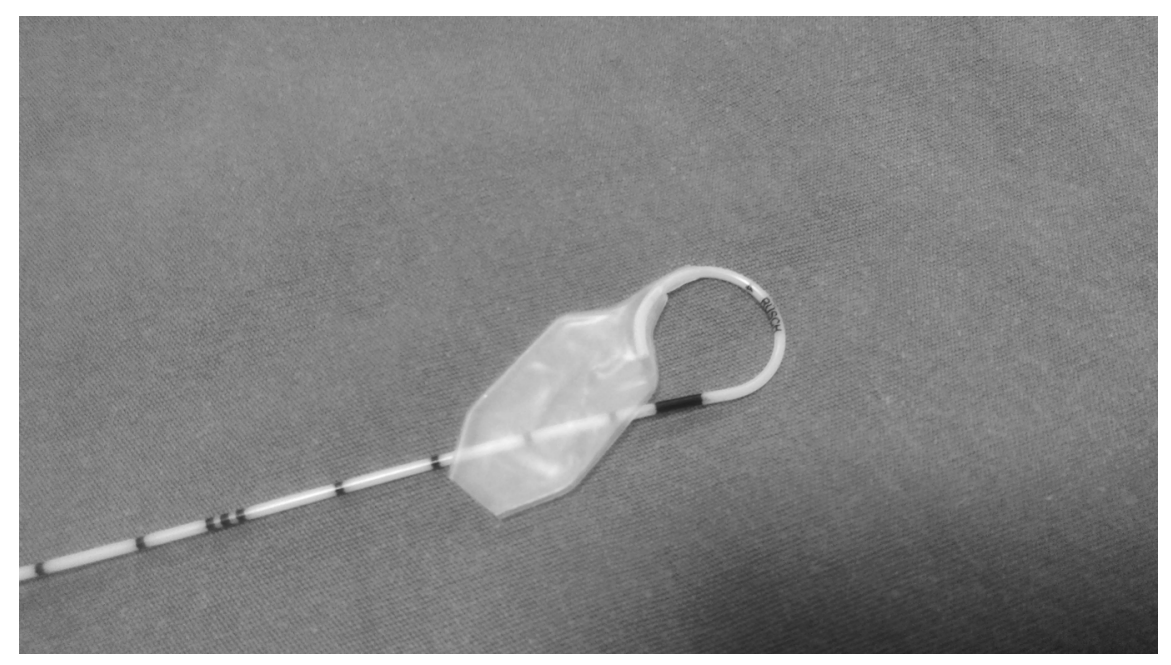

